\title{
Toothpick Colon Injury Mimicking Colonic Diverticulitis
}

\author{
Min Hyeong Jo, Hwan Namgung \\ Department of Surgery, Dankook University College of Medicine, Cheonan, Korea
}

\begin{abstract}
Although toothpick ingestion is rare, it can lead to fatal complications in the gastrointestinal tract. Diagnosing toothpick ingestion is difficult because most patients do not recall swallowing one. We report 2 cases of toothpick-ingestion-induced colon injury, mimicking diverticulitis. The first patient was a 47 -year-old male who had received conservative treatment under the impression of his having diverticulitis in the cecum. Ultrasonography revealed a linear foreign body in the right lower abdomen; a subsequent laparoscopic examination revealed inflammation around the cecum, but no evidence of bowel perforation. A thorough investigation revealed a toothpick embedded in the subcutaneous fat and muscle layer of the lower abdominal wall; we removed it. The second patient was a 56-year-old male who had received conservative treatment under the impression of his having diverticulitis in the sigmoid colon. An explorative laparotomy revealed a toothpick piercing the sigmoid colon; we performed an anterior resection. Both patients were discharged without postoperative complications.
\end{abstract}

Keywords: Toothpick; Colon; Injury

\section{INTRODUCTION}

Toothpick ingestion is difficult to diagnose because most patients do not recall swallowing one, symptoms and physical examinations are nonspecific, and a toothpick has a radiolucent character. Therefore, suspicion is needed to diagnose toothpick ingestion. We report the cases of 2 patients who had been diagnosed as having colonic diverticulitis and had received several weeks of conservative treatment, but were later found to be suffering from toothpick-induced colon injury.

\section{CASE REPORTS}

\section{Case 1}

The study was approved by the Institutional Review Board of the Dankook University Hospital and the informed consent was

Received: November 23, 2017 - Accepted: April 23, 2018

Correspondence to: Hwan Namgung, M.D.

Departments of Surgery, Dankook University College of Medicine, 119 Dandae-ro, Dongnam-gu, Cheonan 31116, Korea

Tel: +82-41-550-3929, Fax: +82-41-565-6167

E-mail: gsnamgung@dankook.ac.kr

ORCID code: https://orcid.org/0000-0002-0783-7642

(C) 2018 The Korean Society of Coloproctology

This is an open-access article distributed under the terms of the Creative Commons Attribution NonCommercial License (http://creativecommons.org/licenses/by-nc/4.0) which permits unrestricted noncommercial use, distribution, and reproduction in any medium, provided the original work is properly cited. waived. A 47-year-old male was referred to our Emergency Department with recurrent pain in the right lower abdominal pain. He had received conservative treatment for 3 weeks at a private clinic under the impression that he was suffering from diverticulitis in the cecum. He had a history of pulmonary tuberculosis and hepatitis B and had undergone an appendectomy 10 years earlier. Physical examination revealed normal vital signs and mild tenderness of the right lower quadrant of the abdomen. Routine blood tests were normal except for elevated C-reactive protein (CRP) (6.33 mg/dL). Abdominopelvic computed tomography (CT) showed cecal wall thickening with pericecal fat infiltration and scanty ascites in the pelvis, suggestive of diverticulitis in the cecum (Fig. 1A). The patient was hospitalized in the Department of Gastroenterology and received conservative treatment for 2 weeks. The symptoms were much improved, but mild abdominal pain still remained, so abdominal ultrasonography (USG) was performed and showed a linear foreign body in the right lower abdominal wall (Fig. 1B); we decided to perform a laparoscopic examination. That examination revealed no evidence of fluid collection or bowel contents in the abdominal cavity, but it did reveal adhesion between the abdominal wall, the omentum, and the cecum (Fig. 1C). We performed adhesiolysis, but at first, we could not find the foreign body or any evidence of bowel perforation (Fig. 1D, E). Then, using a right lower abdominal skin incision, we found a toothpick embedded in the subcutaneous fat and muscle layer and removed it (Fig. 1F). Because of concerns about 


\section{Annals of Toothpick Colon Injury Mimicking Colonic Diverticulitis \\ Coloproctology Min Hyeong Jo and Hwan Namgung}

wound infection, the wound was left open for delayed closure. The patient was discharged seven days after the surgery without complications.

\section{Case 2}

A 56-year-old male was referred to the Outpatient Department complaining of left lower abdominal pain that had been present for 3 weeks. He had received conservative treatment for 10 days at a private clinic under the impression of his having diverticulitis in the sigmoid colon. Because his symptoms had not improved very much, he visited our hospital for further evaluation. He had been diagnosed with hypertension 20 years earlier and had no history
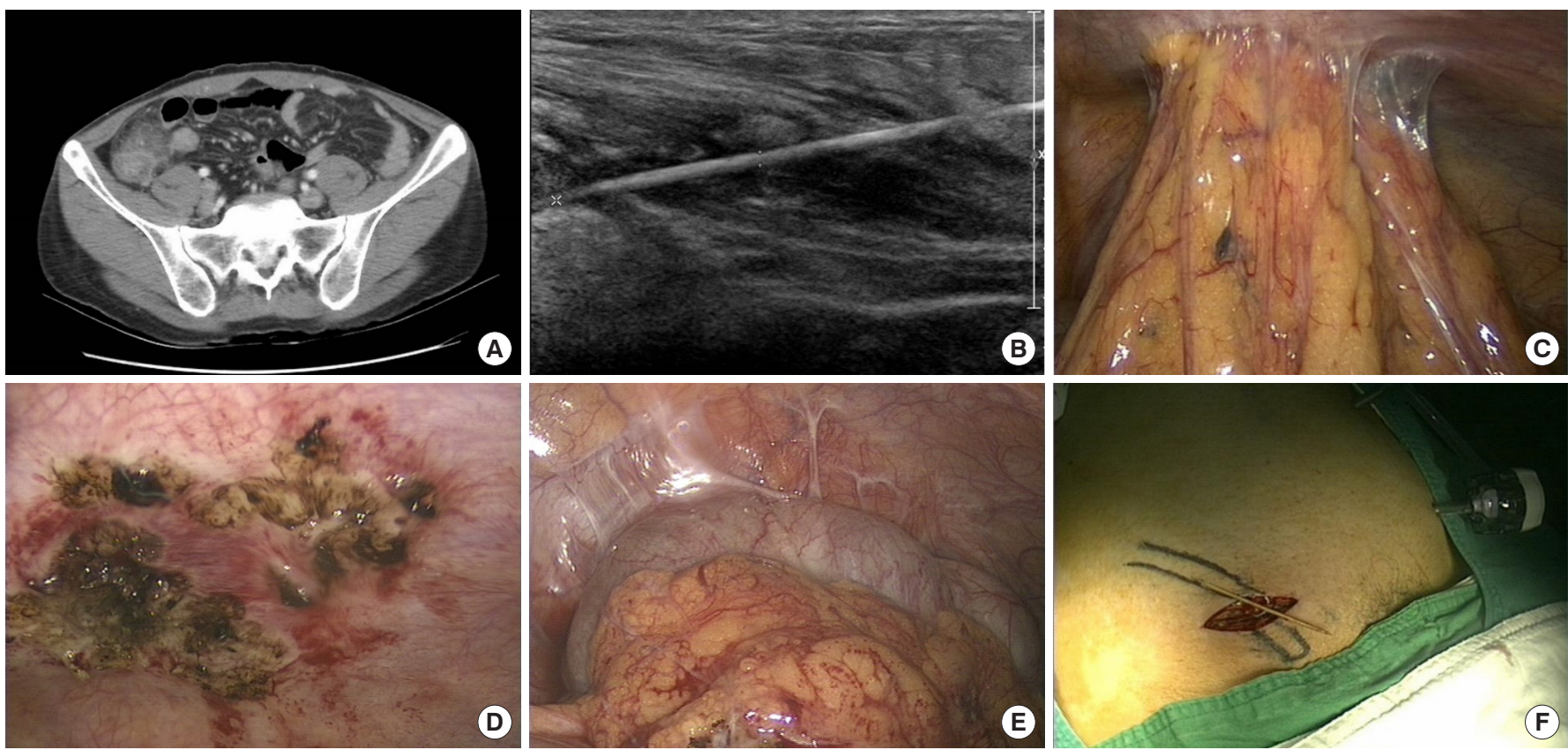

Fig. 1. (A) Computed tomography finding showing cecal wall thickening with inflammation. (B) Ultrasonography finding showing a linear foreign body in the right lower abdominal wall. (C) Laparoscopic finding showing adhesion between the abdominal wall, the omentum, and the cecum. (D, E) Laparoscopic finding: after adhesiolysis, we were unable to find a foreign body or any evidence of bowel perforation. (F) Operative finding: the toothpick was embedded in the subcutaneous layer of the right lower abdominal wall.

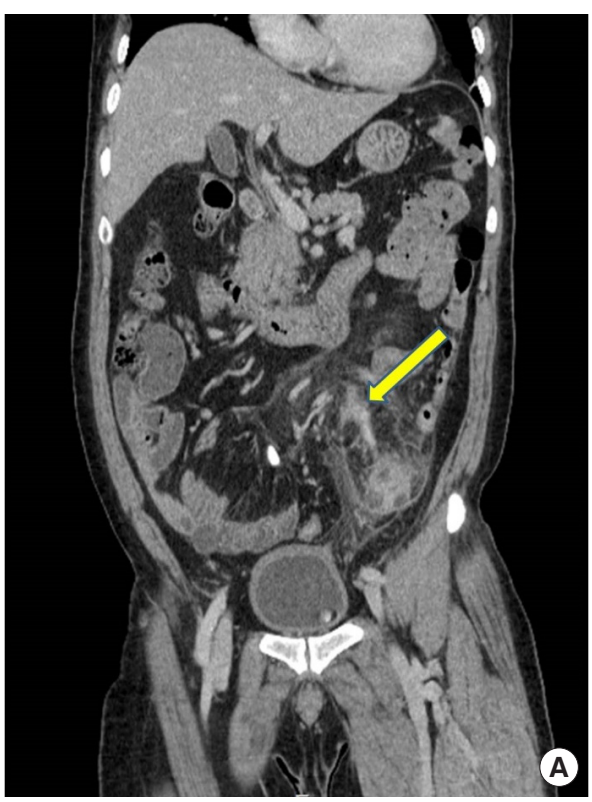

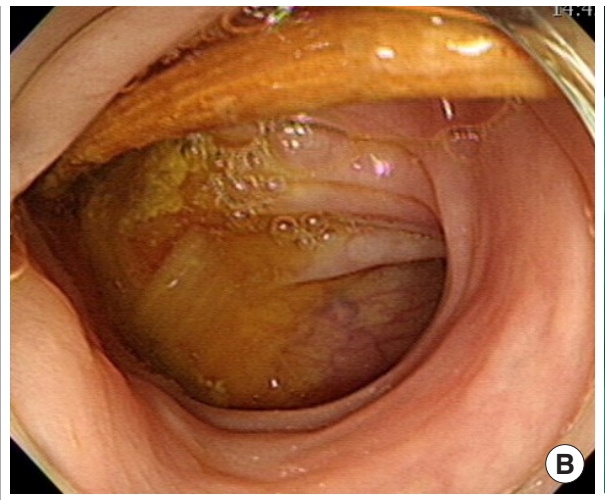

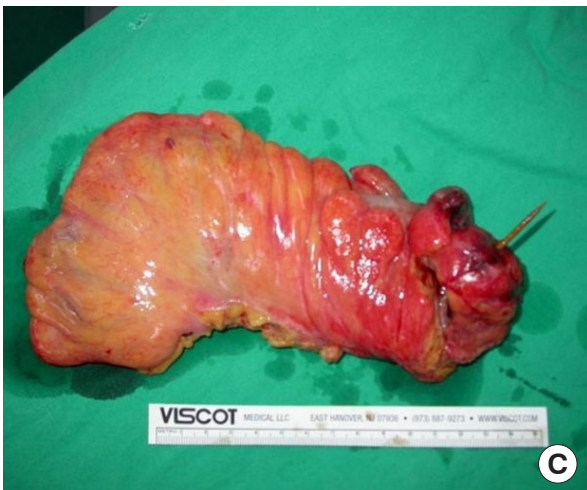

Fig. 2. (A) Computed tomography finding suggesting diverticulitis in the sigmoid colon with a suspicious fistulous tract (arrow). (B) Colonoscopy finding: a toothpick is shown at the sigmoid colon, but it was missed at that time. (C) Resected sigmoid colon showing a toothpick piercing the sigmoid colon. 
of surgery. The physical examination revealed normal vital signs and mild tenderness of the left lower quadrant of the abdomen. Routine blood tests were normal without leukocytosis or elevated CRP. Abdominopelvic CT showed wall thickening of the sigmoid colon and pericolic fat infiltration with a linear line in the left lower mesentery, suggestive of diverticulitis in the sigmoid colon with a suspicious fistulous tract (Fig. 2A). Colonoscopy revealed erythematous mucosal inflammation in the sigmoid colon. The toothpick was shown at the sigmoid colon, but was missed at that time (Fig. 2B). The patient received conservative treatment, and a follow-up CT was done 10 days after admission. In the follow-up CT, a linear high-density structure in the mesentery connected to the sigmoid colon was seen and was suspected of being a foreign body penetrating the sigmoid colon, so the patient was referred to the Surgery Department for operative management. An explolaparotomy revealed a toothpick piercing the sigmoid colon with pericolic inflammation, so an anterior resection was performed (Fig. 2C). The patient was discharged nine days after the surgery without complications.

\section{DISCUSSION}

Foreign body ingestion can cause various gastrointestinal (GI) complications, including perforation, bleeding, obstruction and even sepsis [1-3]. Most ingested foreign bodies pass through the GI tract without any complications, so GI perforation is rare, occurring in $<1 \%$ of patients $[1,4]$. However, the ingestion of a toothpick has a higher risk of complications because of its being sharp and pointed on both ends [1].

Several risk factors are associated with toothpick ingestion. These include being a child or elderly person, a dull palatal sensation due to dentures, alcohol intoxication, and habitual chewing of toothpicks [5]. The common sites of injury are the duodenum, ileocecal area, the appendix, splenic flexure and recto-sigmoid areas. This is attributed to abrupt physiological luminal narrowing, bowel angulation and the transition from a mobile to a more fixed portion of the bowel $[1,4,6]$. After the toothpick has perforated the bowel wall, it can migrate into adjacent or distant organs, including the liver, pancreas, great vessels and heart $[1,4]$. The preoperative diagnosis of toothpick ingestion is difficult because only $12 \%$ of patients recall the episode of toothpick ingestion [1]. If the patient is aware of toothpick ingestion, endoscopy is the most effective technique for identifying and removing a foreign body embedded in the proximal or distal GI tract [7]. In patients for whom no indications pointing toward toothpick ingestion can be found, CT is a useful technique for identifying intestinal wall thickening, regional fatty infiltration, localized pneumoperitoneum or abscess [1]. Although CT can identify wooden objects, the identification of wooden toothpicks with certainty is difficult due to their radiolucent nature and small diameters [5]. USG or magnetic resonance imaging (MRI) is a better diagnostic tool for detecting wooden foreign bodies [5, 8].

In our cases, the toothpick was not recognized initially. In the first case, the toothpick was clearly identified using USG, but was not prominent on the CT scan. In the second case, a retrospective review showed that colonoscopy had revealed a toothpick-like structure, but the toothpick embedded in the colon was not readily recognized because bowel preparation was insufficient and no suspicions of toothpick ingestion were present. In both cases, the initial impression of the patients was diverticulitis of the colon, which shows response to antibiotic therapy. However, the symptoms did not improve sufficiently as a result of such therapy, so a follow-up CT scan was done; the results showed no significant changes from the previous CT study. If other imaging studies, such as USG and MRI, had been done earlier, a correct diagnosis and plan of management could have been achieved sooner [5].

In conclusion, a strong suspicion is needed in the diagnosis of toothpick ingestion. We should always keep in mind the possibility of foreign-body ingestion when patients complain of relapsing abdominal pain of unknown origin.

\section{CONFLICT OF INTEREST}

No potential conflict of interest relevant to this article was reported.

\section{REFERENCES}

1. Zouros E, Oikonomou D, Theoharis G, Bantias C, Papadimitropoulos K. Perforation of the cecum by a toothpick: report of a case and review of the literature. J Emerg Med 2014;47:e133-7.

2. Li SF, Ender K. Toothpick injury mimicking renal colic: case report and systematic review. J Emerg Med 2002;23:35-8.

3. Chichom-Mefire A. Perforation of the splenic flexure of the colon by an ingested wooden toothpick. J Surg Case Rep 2015;2015.

4. Goh BK, Chow PK, Quah HM, Ong HS, Eu KW, Ooi LL, et al. Perforation of the gastrointestinal tract secondary to ingestion of foreign bodies. World J Surg 2006;30:372-7.

5. Sealock RJ, Sabounchi S, Graham DY. Toothpick perforation of the intestines presenting as recurrent abdominal pain: possible roles of abdominal ultrasound and MRI. Clin Med Insights Case Rep 2013;6:131-5.

6. Pan CC, Wang CP, Huang JJ, Chen WK, Yang HR. Intestinal perforation after the incidental ingestion of a fishhook. J Emerg Med 2010;38:e45-8.

7. Steinbach C, Stockmann M, Jara M, Bednarsch J, Lock JF. Accidentally ingested toothpicks causing severe gastrointestinal injury: a practical guideline for diagnosis and therapy based on 136 case reports. World J Surg. 2014;38:371-7.

8. Orlinsky M, Knittel P, Feit T, Chan L, Mandavia D. The comparative accuracy of radiolucent foreign body detection using ultrasonography. Am J Emerg Med 2000;18:401-3. 